\title{
ENHANCED VEHICLE IDENTIFICATION UTILIZING SENSOR FUSION AND STATISTICAL ALGORITHMS
}

\author{
Stephane Roussel \\ California Polytechnic State University \\ San Luis Obispo, CA, USA \\ email: sroussel@calpoly.edu
}

\author{
Hemanth Porumamilla \\ California Polytechnic State University \\ San Luis Obispo, CA, USA \\ email: hporumam@calpoly.edu
}

\author{
Charles Birdsong \\ California Polytechnic State University \\ San Luis Obispo, CA, USA \\ email: cbirdson@calpoly.edu
}

\author{
Peter Schuster \\ California Polytechnic State University \\ San Luis Obispo, CA, USA \\ email: pschuste@calpoly.edu
}

\author{
Christopher Clark \\ California Polytechnic State University \\ San Luis Obispo, CA, USA \\ email: cmclark@csc.calpoly.edu
}

\begin{abstract}
Several studies in the area of vehicle detection and identification involve the use of probabilistic analysis and sensor fusion. While several sensors utilized for identifying vehicle presence and proximity have been researched, their effectiveness in identifying vehicle types has remained inadequate. This study presents the utilization of an ultrasonic sensor coupled with a magnetic sensor and the development of statistical algorithms to overcome this limitation.
\end{abstract}

Mathematical models of both the ultrasonic and magnetic sensors were constructed to first understand the intrinsic characteristics of the individual sensors and also to provide a means of simulating the performance of the combined sensor system and to facilitate algorithm development. Preliminary algorithms that utilized this sensor fusion were developed to make inferences relating to vehicle proximity as well as type. It was noticed that while it helped alleviate the limitations of the individual sensors, the algorithm was affected by high occurrences of false positives. Also, since sensors carry only partial information about the surrounding environment and their measured quantities are partially corrupted with noise, probabilistic techniques were employed to extend the preliminary algorithms to include these sensor characteristics. These statistical techniques were utilized to reconstruct partial state information provided by the sensors and to also filter noisy measurement data. This probabilistic approach helped to effectively utilize the advantages of sensor fusion to further enhance the reliability of inferences made on vehicle identification.

In summary, the study investigated the enhancement of vehicle identification through the use of sensor fusion and statistical techniques. The algorithms developed showed encouraging results in alleviating the occurrences of false positive inferences. One of the several applications of this study is in the use of ultrasonic-magnetic sensor combination for advanced traffic monitoring such as smart toll booths.

\section{INTRODUCTION}

The use of probabilistic techniques enhances the credibility of conclusions made through data analysis. However, a more basic and necessary requirement for vehicle detection is the use of suitable sensors. Literature review suggested the suitability of ultrasonic sensors and magnetic sensors [1-14]. Based on this information these two sensors were selected for incorporation into the test matrix. In the following paragraphs, information on the specific type of sensors that were used and fundamental testing (analytical and experimental) that was carried out to understand their working principles is discussed.

\section{Ultrasonic Sensors}

Ultrasonic sensors have been used in the past for detection and distance measurement in automotive applications $[1,2]$. In these studies a piezoelectric ultrasonic transducer emitting an inaudible sound wave was utilized. These ultrasonic transducers provide distance measurement based on the time taken to receive an echo from a transmitted signal. A similar ultrasonic 
sensor (Fig. 1) was used for detection of vehicle presence. Modeling of the ultrasonic sensor focused on range and accuracy of measurement for the application in hand (Fig 1). This specific sensor was chosen due to its narrow beam characteristic (Fig. 1), which has the ability to reject false detection caused by wide angle reflection, typical of many ultrasonic sensors [3].
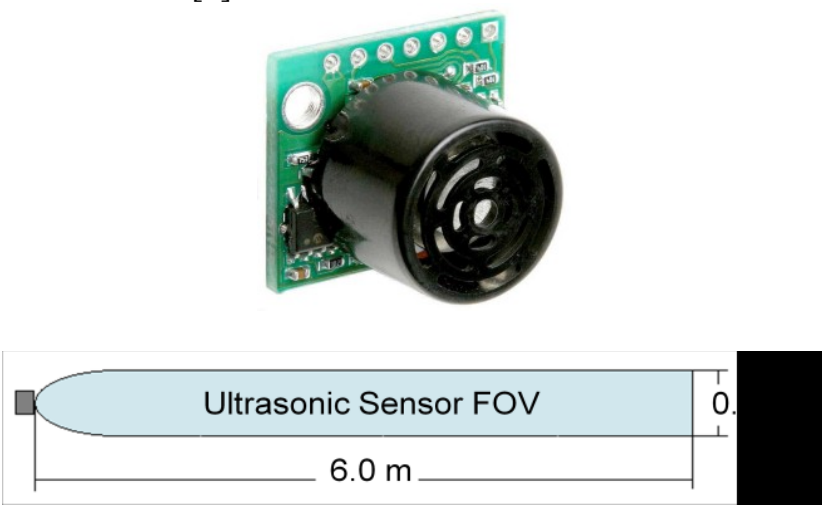

Fig 1. MaxBotix ultrasonic sensor with corresponding field of view

\section{Magnetic Sensors}

In the recent past, extensive research in the area of magnetic sensors has resulted in the invention of different types of magnetic sensors that are capable of measuring magnetic field strengths in the $\mu$ Gauss range through the range of several millions of Gauss [4]. One such magnetic sensor is of a magneto-resistive type as shown in Fig. 1. Typical magnetoresistive sensors are low cost, high sensitivity magnetic devices with a measurement range from several $\mu$ Gauss to tens of Gauss [5]. In addition, their small size and resilience to harsh environments have led to their extensive use in varied applications such as navigational systems [6], [7], traffic surveillance [8], [9], and vehicle detection [7], [10], and [11]. Some research has also been conducted on the use of magnetic sensors onboard vehicles for proximity and blind spot detection [12], [13]. Based on this background information, a similar magneto-resistive type sensor was utilized in this project for the purpose of vehicle type detection.
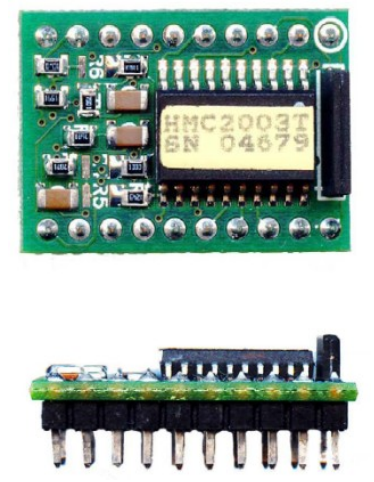

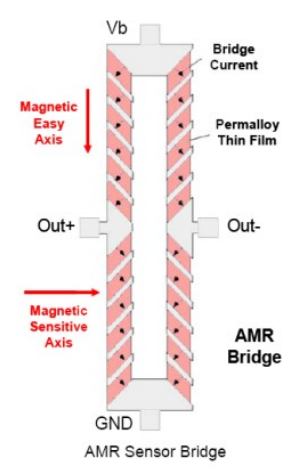

A detailed description of research on the magnetic sensors done by the authors was submitted for publication in [14] and has been briefly described here as background information. In this project the magnetic sensor was mounted on the lateral side of a test vehicle as shown in Fig. 3. The magnetic signatures of vehicles passing by the test vehicle were captured (Fig. 3). A high fidelity 3-D multi-dipole mathematical model was developed for typical on road vehicles (Fig. 4) and the model parameters were tuned such that the simulation response corroborated the experimental findings (Figs. 5 and 6).

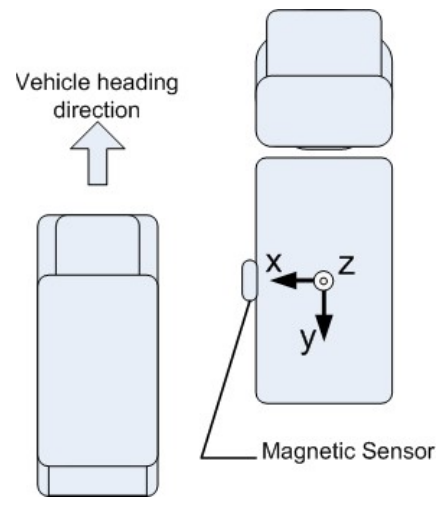

Fig 3. Road test configuration

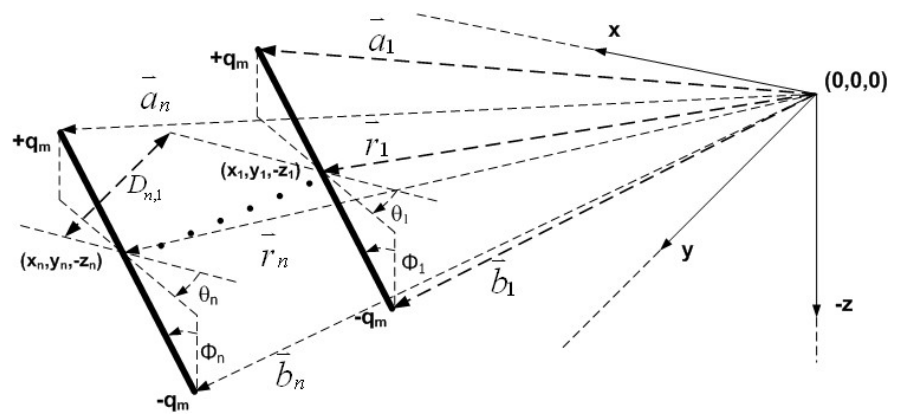

Fig 4. Magnetic dipole in 3-D space
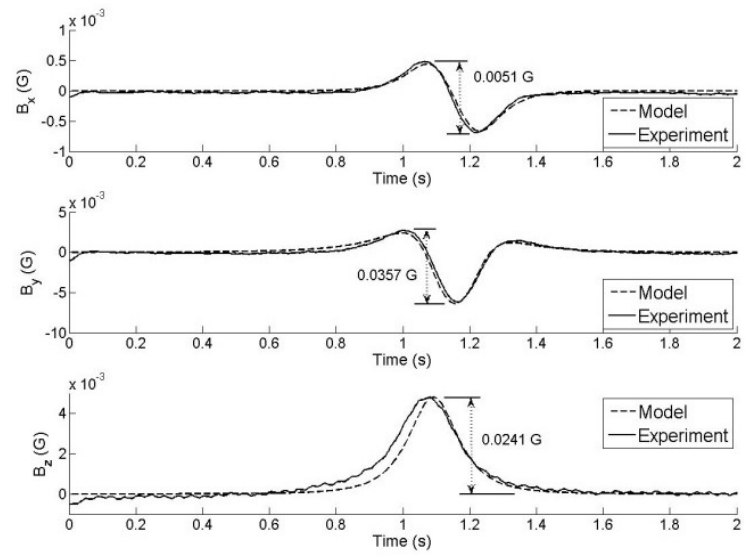

Fig 5. Simulation vs. experiment (passenger car)

Fig 2. HMC 2003 Magneto-resistive sensor 

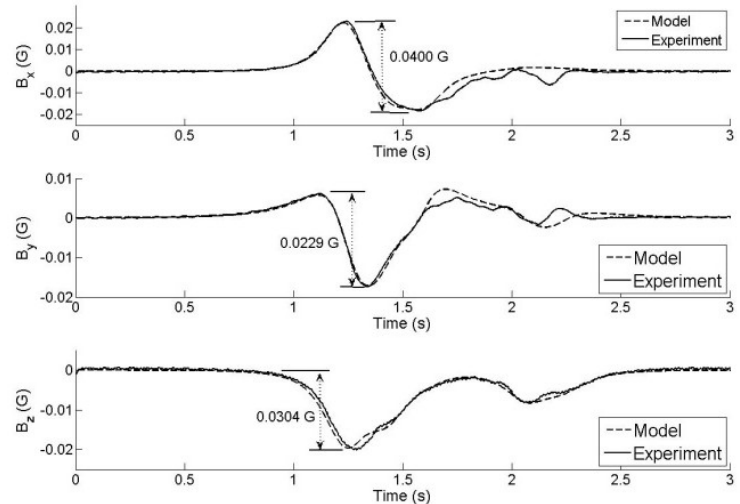

Fig 6. Simulation vs. experiment (large truck)

Vehicle identification using magnetic sensors was achieved by applying suitable mathematical functions to the magnetic field strengths in Figs. 5 and 6. The result is shown in Fig. 7.

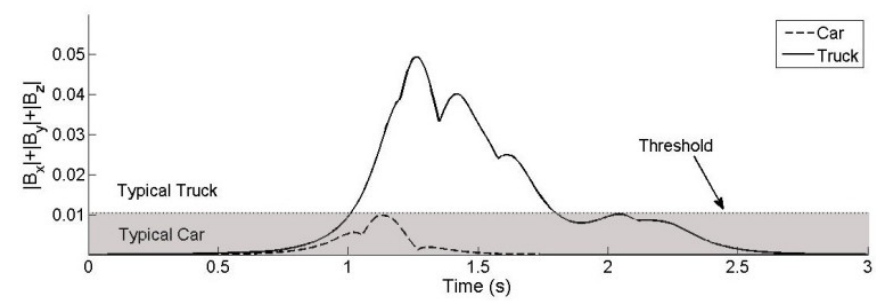

Fig 7. Threshold detection (car and truck)

\section{BASIC CONCEPTS IN PROBABILITY}

For this application, voltage measurements taken from individual sensors are treated as random variables. Let the random variable (sensor measurement) be denoted by the variable $Z$ and the specific sensor reading at time $t$ be denoted as $Z_{t}$. These random variables can take on multiple values, and they do so according to specific probabilistic laws. Probabilistic law can be defined for sensor measurements for specific applications, such as vehicle detection and is explained in the next section.

The probabilistic nature of the sensor's behavior is modeled using, probability density functions (PDFs). The sensor probability density functions are that of the onedimensional normal distribution with mean $\mu$ and variance $\sigma^{2}$. These PDFs of normal distribution are given by the following Gaussian function:

$$
p\left(z_{t}\right)=\left(2 \pi \sigma^{2}\right)^{-\frac{1}{2}} \exp \left\{\frac{-(z-\mu)^{2}}{2 \sigma^{2}}\right\}
$$

where, $p(z)$ represents the likelihood of a sensor measurement $Z$ with an expected value of $\mu$ and the variability of the sensor measurement represented by $\sigma^{2}$. The density function for the ultrasonic sensor and magnetic sensor are derived later in "Statistical Sensor Modeling" and used to attain the likelihood of vehicles being present around the host vehicle while taking into account false targets.

The information from an individual sensor can easily be compared with data from other sensors when applying a probabilistic approach for multiple sensors; this process is called joint distribution. Joint distribution describes the probability that the random variable $X=x$ and that $Y=y$. If $X$ and $Y$ are independent the joint distribution is given to be

$$
p(x, y)=p(x) p(y)
$$

Joint distribution is essential for multi-sensor fusion in vehicle detection because presence of an object and its type cannot be positively identified with a single sensor. The above equation can be used to integrate multiple sensors because the information of each sensor is independent of the other. Figures 8 and 9 show the results of joint distribution between two sensor belief curves. If two sensors are in agreement, the joint likelihood has a unique mode at the estimated state variable; however, when the sensors are in disagreement, the joint likelihood is bimodal and has a low likelihood at the estimated state variable. This idea of joint distribution can easily be applied to the ultrasonic sensors and magnetic sensors to check for the presence of an object and to ascertain if the object is a vehicle.

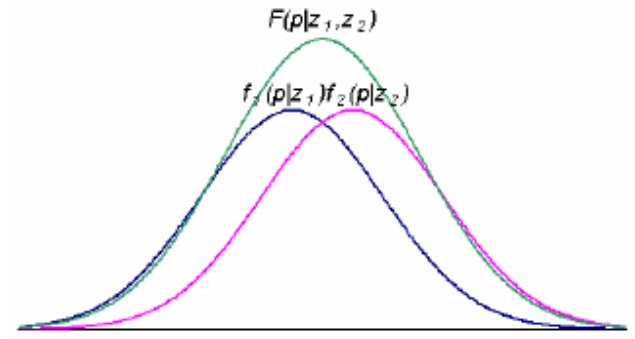

Fig 8. Joint distribution when in agreement [15]

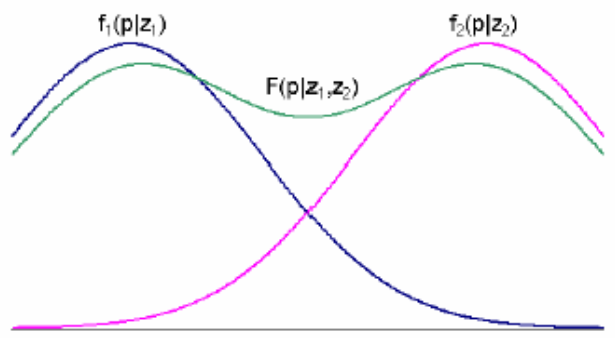

Fig. 9. Joint distribution when in disagreement [15]

Often, random variables carry information about other random variables and thus are not independent. The joint distribution of the two random variables is then called conditional probability, which can be stated as the probability of $X=x$ being true when $Y=y$ is true. 
Conditional probability can be used to describe the behavior of an individual sensor or the likelihood of an event by assuming that the measurement at time $t$ is dependent on the measurement at $t-1$. Conditional probabilities can be examined by a method called Bayes rule (3).

$$
p(x \mid y)=\frac{p(y \mid x) p(x)}{p(y)}
$$

"If $x$ is a quantity that we would like to infer from $y$, the probability $p(x)$ will be referred to as prior probability distribution, and $y$ is called the sensor measurement data. The distribution $p(x)$ summarizes the knowledge we have regarding $X$ prior to incorporating the data $y$. The probability $p(x \mid y)$ is called the posterior probability distribution over $X$. This method provides a convenient way to compute posterior conditional probability $p(x \mid y)$ using the "inverse" conditional probability $p(y \mid x)$ along with the prior probability $p(x)$.In other words, if we are interested in inferring a quantity $x$ from sensor data y, Bayes rule allows us to do so through the inverse probability, which specifies the probability of the data $y$ assuming that $x$ was the case." [16]

The following section expands on the basic concepts of probability and explains how Bayes rule can be modified into a filter to reject sensor noise. Furthermore, this filter is modified to handle information from the ultrasonic and magnetic sensors and play an active role in the purposed vehicle detection algorithm.

\section{BAYESIAN FILTERING}

Raw data from sensors is corrupted by process noise and anomalies due to environmental influences as seen in Fig. 10. Bayesian filters can be created to filter noisy or partial sensor data using the basic concepts in probability from the previous section $[16,17]$. A Bayesian filter is a recursive state estimation model with the ability to output the likelihood of an event occurring. The state of the surroundings around sensors cannot be measured directly; however, the likelihood of the state can be inferred through sensor data and a Bayesian filter. The filter is completed in two steps: the prediction step and correction step.

Prediction Step: At each time update, the state is predicted according to the following update rule.

$$
\operatorname{Bel}^{-}\left(x_{t}\right)=\int p\left(x_{t} \mid x_{t-1}\right) \operatorname{Bel}\left(x_{t-1}\right) d x_{t-1}
$$

The predicted belief of the state variable at time $t$, which is $\mathrm{Bel}^{-}\left(x_{t}\right)$ is represented by the integral or sum of the product of two distributions: the prior distribution $\operatorname{Bel}\left(x_{t-1}\right)$ and a predicted belief based on the prior distribution. The term $p\left(x_{t} \mid x_{t-1}\right)$ describes the system dynamics, which ascertains how the state of the system changes over time. This term predicts the likelihood of the system state based on the last belief. The prediction parameters are described in the following section.

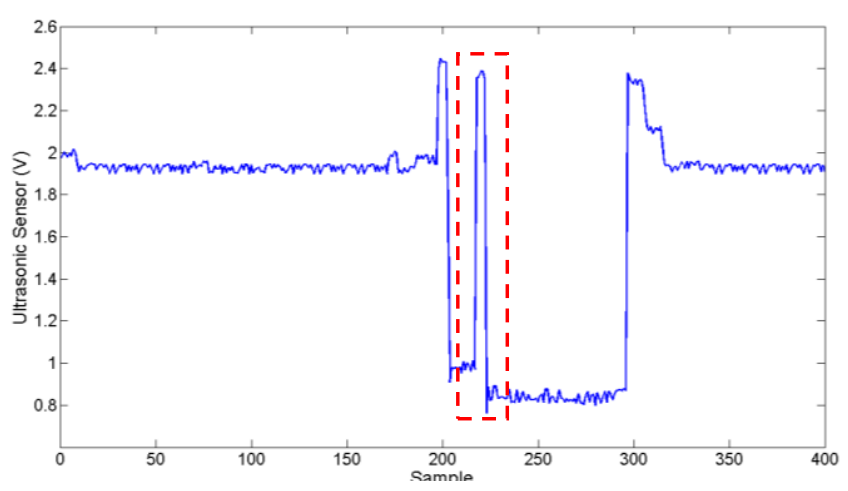

Fig 10. Raw ultrasonic data with noise

Correction Step: Whenever new sensor information $z_{t}$ is received, the measurement is used to correct the predicted belief using the observation.

$$
\operatorname{Bel}\left(x_{t}\right)=\eta p\left(z_{t} \mid x_{t}\right) \operatorname{Bel}\left(x_{t}\right)
$$

The term $p\left(z_{t} \mid x_{t}\right)$ is the perceptual model that describes the likelihood of making observation $z_{t}$ given that a state variable is equal to $x_{t}$. For location estimation, the perceptual model is usually considered a property of a given sensor technology. It depends on the types and positions of these sensors and captures a sensor's error characteristics. The term $\eta$ is a normalizing constant which ensures that the posterior over the entire state space sums up to one. This constant is discussed in more detail in the following section.

\section{BAYES FILTER ALGORITHM}

Bayesian filtering can be directly applied to the sensors for the purposes of vehicle detection. To clearly explain how the Bayes filter algorithm is developed; let's consider only the ultrasonic sensor with the state variable of interest $x$ being the presence of an object. This procedure will later be expanded to include the magnetic sensor and other state variables. As mentioned in the previous section, the Bayesian filter is completed in two steps: prediction and correction.

Prediction Step: The predicted model for the ultrasonic sensor is based on Theorem of Total Probability. The following equation represents the predicted probability of an object's presence at time $t$ based on the probability of an object's presence at time $t-1$.

$$
p\left(x_{t}^{\prime}\right)=p\left(x_{t}^{\prime} \mid x_{t-1}\right) p\left(x_{t-1}\right)+p\left(x_{t}^{\prime} \mid 7 x_{t-1}\right) p\left(7 x_{t-1}\right)
$$

Here, the terms $p\left(x_{t}^{\prime} \mid x_{t-1}\right)$ and $p\left(x_{t}^{\prime} \mid 7 x_{t-1}\right)$ describe the predicted probability that an object is present at time $t$ based on the probability that an object is present at time $t-1$ and the probability that an object is absent at time $t-1$ respectively. In detecting an object's presence, this conditional probability is the motion model where the vehicle might be at time $t$, given its location at $x_{t-1}$. 
Correction Step: Using the information from the prediction step, the likelihood of a vehicle's presence $p(x)$ and a vehicle's absence $p(7 x)$ are evaluated using the correction step. The correction step of the algorithm is represented by:- of the algorithm.

$$
\begin{gathered}
p(x)=\eta p\left(z_{t} \mid x_{t}^{\prime}\right) p\left(x_{t}^{\prime}\right) \\
p(7 \mathrm{x})=\eta p\left(z_{t} \mid 7 \mathrm{x}_{\mathrm{t}}^{\prime}\right) p\left(x_{t}^{\prime}\right) p\left(7 \mathrm{x}_{\mathrm{t}}^{\prime}\right) \\
\eta=\left[p\left(z_{t} \mid x_{t}^{\prime}\right) p\left(x_{t}^{\prime}\right)+p\left(z_{t} \mid 7 \mathrm{x}_{\mathrm{t}}^{\prime}\right) p\left(7 \mathrm{x}_{\mathrm{t}}^{\prime}\right)\right]^{-1}
\end{gathered}
$$

where $\eta$ represents the normalizing parameter to ensure the probability of a $p(x)$ and $p(7 x)$ are between 0 and 1.

\section{STATISTICAL SENSOR MODELING}

The Bayesian filter requires specific parameters for both the prediction and correction steps. The following section describes the prediction parameters and correction parameters used for the ultrasonic sensor and magnetic sensor. Data was collected for a vehicle passing scenario as mentioned in the introduction.

\section{Ultrasonic Sensor Model}

The ultrasonic sensor is used to detect the range and presence of an object on the lateral side of a large truck. The prediction and correction models for this sensor can be achieved because this sensor is operating in a specific manner with physical constraints (i.e. lane dimension and vehicle dimensions) and detection frequency (i.e. traffic flow). For this sensor, the state variable of interest is presence of a vehicle. In other words, when the ultrasonic sensor is filtered through the Bayesian filter, the output is the likelihood that a vehicle is present.

To describe the sensors behavior, a perceptual model is required. For this application, the perceptual model was created for the ultrasonic sensor by monitoring the lane on the lateral side of a large truck. As vehicles pass by the sensors, the average distances are recorded and average sensor behavior identified. The sensor behaviors can be modeled as Gaussian distributions and used in the correction step of the filter. The histograms shown below represent the behavior of an ultrasonic sensor when vehicles are present and absent. The average distance of a passing car from this model is about $6.25 \mathrm{ft}$ (sensor voltage of $0.71 \mathrm{~V}$ ). It is important to note that some transmitted signals from the ultrasonic sensor may be reflected off a vehicle's body and not be received by the sensor. This is accounted for in the sensor model by its bimodal nature (i.e. the second mode at Fig. 11 in the distance range 18-21 ft).

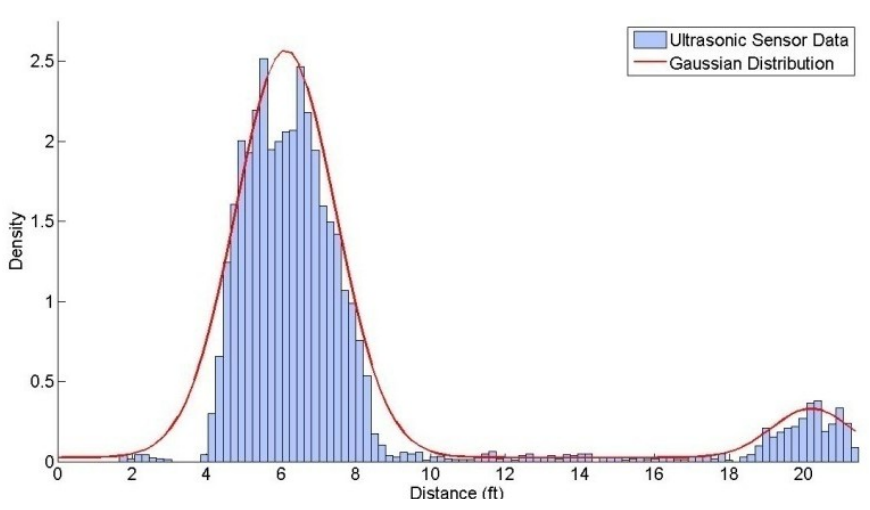

Fig 11. Ultrasonic sensor belief distribution when vehicle present

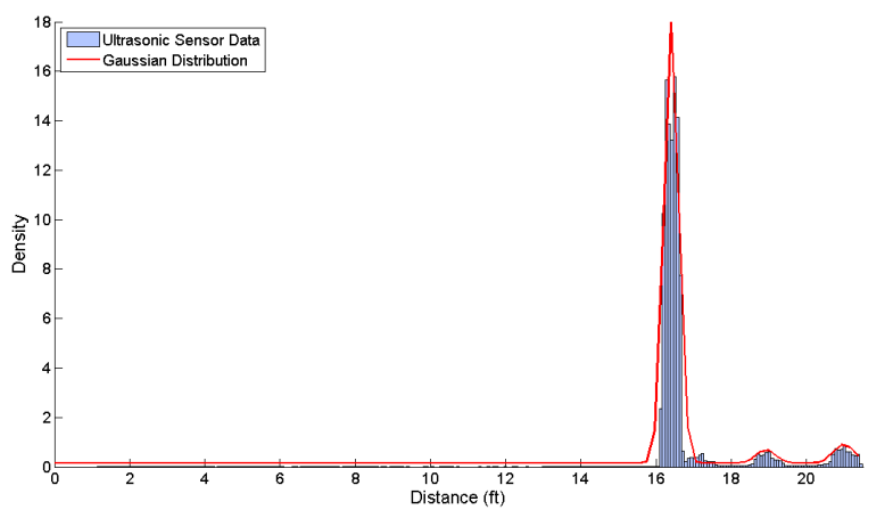

Fig 12. Ultrasonic sensor belief distribution when vehicle absent

\section{Magnetic Sensor Model}

The magnetic sensor was tested in the same manner as the ultrasonic sensor. The magnetic fields in all three axes $(x, y, z)$ were recorded as vehicles passed by the sensor. As mentioned in the "Introduction" section, a math function was used to positively detect vehicle presence (10).

$$
\left|\mathrm{B}_{\mathrm{x}}\right|+\left|\mathrm{B}_{\mathrm{y}}\right|+\left|\mathrm{B}_{\mathrm{z}}\right|
$$

Unlike the ultrasonic sensor when a vehicle passes by a magnetic sensor, the change in sensor voltage is gradual and is not proportional to the vehicle's length. To accurately identify a vehicle's presence from the magnetic sensor, its behavior is modeled as (11) for an incremented range of data rather than frequency.

$$
\frac{\text { Number of "Present" Measurements }}{\text { Total Number of Measurements }}
$$

The behavior for the magnetic data is best described using a two step function and a Gaussian distribution (Fig. 13). The 
same process is followed to describe the magnetic sensor's behavior during the absence of a vehicle.

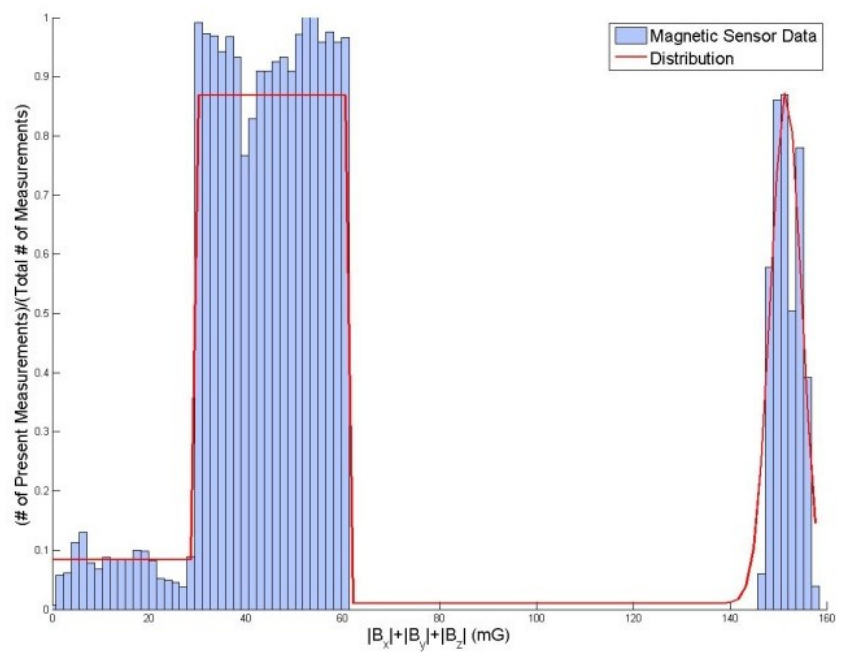

Fig 13. Magnetic sensor belief distribution when vehicle present

\section{Prediction Model}

The prediction step requires the probability that an event will occur at time $t$ based on the previous correction step at time $t-1$. These parameters can be based on a variety of ideas. In the case of predicting the likelihood of a vehicle being present, two modes of prediction are considered. One prediction mode is in effect when a vehicle is detected and the other prediction mode takes effect in between vehicles (the absence of a vehicle). The first prediction step takes into account the number of "present" measurements taken by the ultrasonic sensor when a vehicle passes; the number of "present" samples varies with the physical length of a vehicle and its relative velocity. As the number of "present" measurements increases, the predicted probability of a vehicle being present in the next sensor measurement will decrease. This process can be further refined by integrating the magnetic sensor to identify the vehicle type allowing adjustments in the number of predicted "present" measurements based on vehicle length. The same approach mentioned is used for the prediction parameter when a vehicle is absent; however, the number of "absent" measurements is based off of vehicle frequency or traffic flow. As traffic flow increases, the likelihood of a vehicle being absent in the next "absent" measurement will decrease. Figure 14 shows the ultrasonic sensor measurement and predicted likelihood when a vehicle is present and absent.
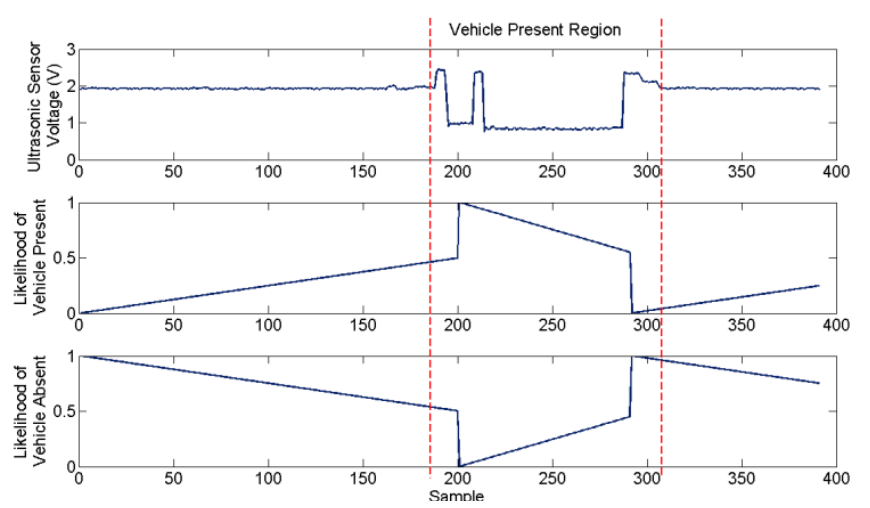

Fig 14. Ultrasonic sensor predictive model

\section{Correction Model}

The correction step uses the sensor models to make correction in the predicted measurements. With the ultrasonic and magnetic sensor models and a prediction model, the probability of an object being present and being a specific type can now be identified using equations $(8-10)$. The results for this prediction and correction methods being applied to both individual sensor case and sensor fusion case are presented in the next section.

\section{BAYESIAN FILTER RESULTS (INDIVIDUAL SENSORS)}

The Bayesian filter is complete with both the prediction and correction models produced above as shown in the following schematic (Fig. 15). The following shows the resulting behavior of the Bayesian filters for the ultrasonic sensor and magnetic sensor.

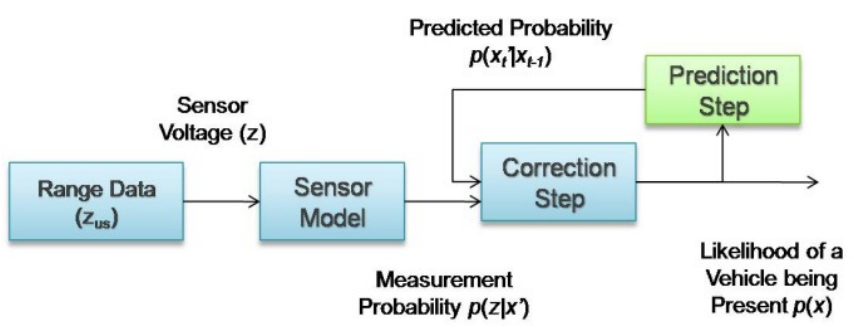

Fig 15. Bayesian filter algorithm (individual sensor)

The individual sensor data, the predictive model, and the corrective model work together to output a belief that a vehicle is present as vehicles pass the ultrasonic sensor. It can be seen in Fig. 16 that the noise in the ultrasonic sensor, such as the large spike at time step 220 has little effect on the belief that a vehicle is present. The same procedure is used for the magnetic sensor (Fig. 17). With this Bayesian filter, the uncertainties that arise from partial and noisy ultrasonic data are accounted for and the belief of a vehicle's presence can be evaluated to make decisions in vehicle identification. 


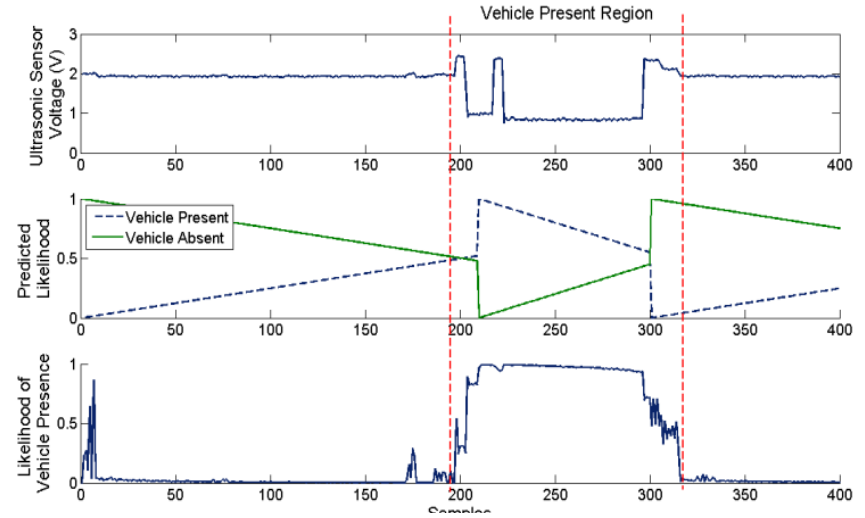

Fig 16. Likelihood of vehicle presence (ultrasonic sensor)
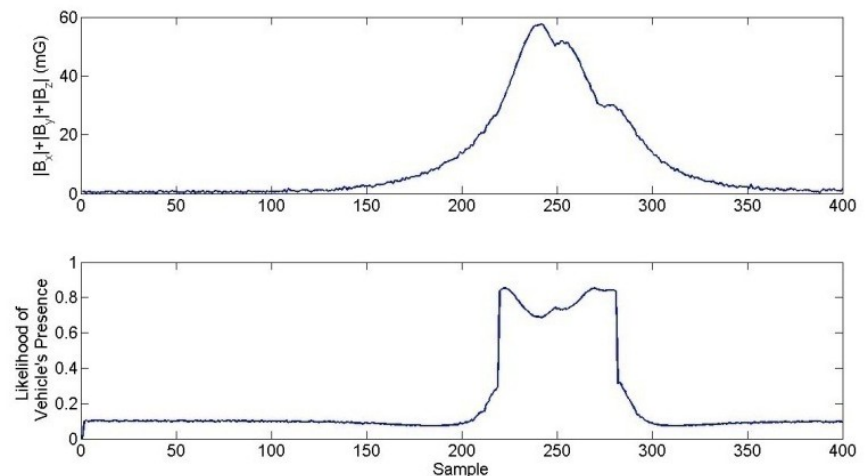

Fig 17. Likelihood of vehicle presence (magnetic sensor)

\section{SENSOR FUSION}

The outputs from the Bayesian filter only represent the belief of individual sensors. Thus, a joint probabilistic method is required to "fuse" this information together (Fig. 18). If the ultrasonic sensor is represented as $S_{1}$ and the magnetic sensor as $S_{2}$, the joint belief distribution can be represented as:

$$
p\left(x \mid z_{S 1}, z_{S 2}\right)=p\left(x \mid z_{S 1}\right) p\left(x \mid z_{S 2}\right)
$$

where $-x$ and $y$ represent independent state variables for the sensors and $z$ represents the joint distribution.

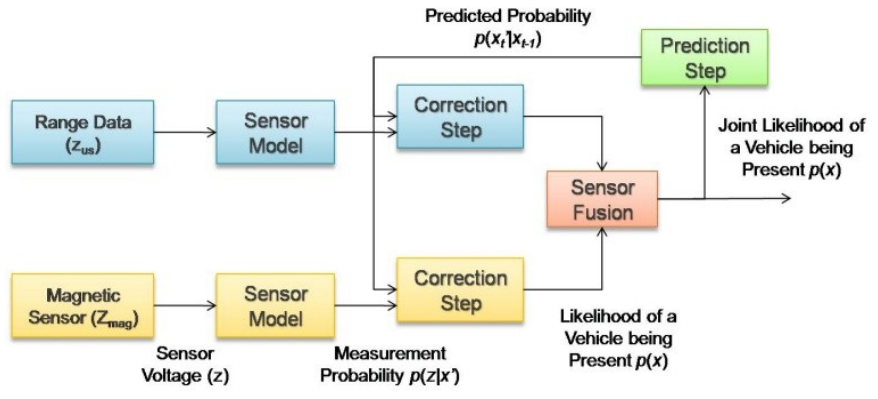

Fig 18. Bayesian filter algorithm (multiple sensors)
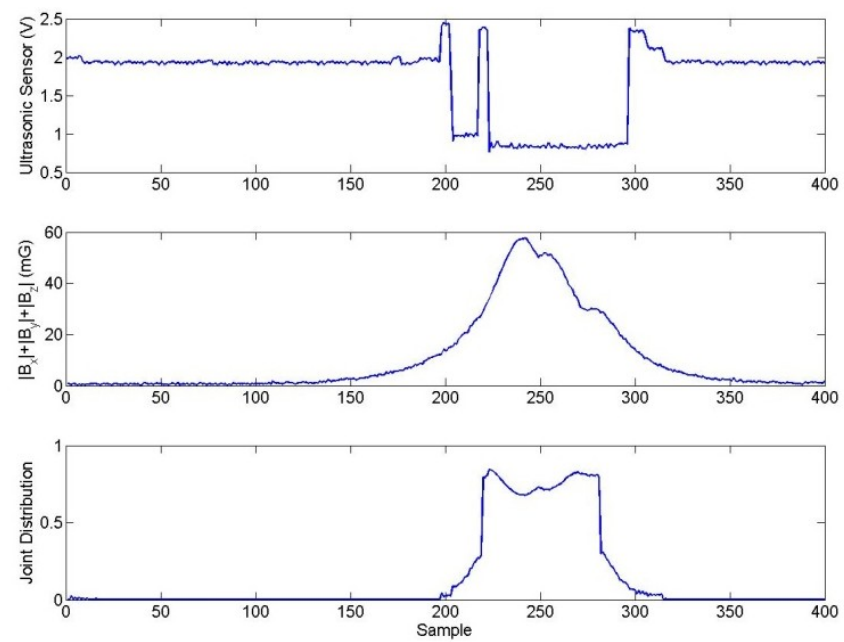

Fig 19. Joint likelihood of vehicle presence
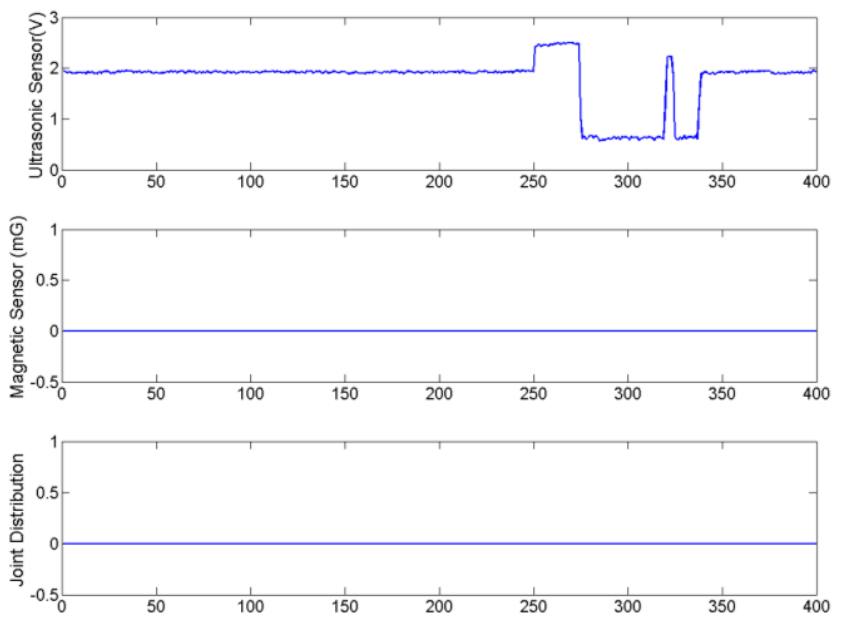

Figure 20. Joint likelihood of a non-metallic object

Figures 19 and 20 show the detection of two objects. In figure 19 the joint likelihood being high (about 0.8) suggests the presence of a vehicle. On the other hand, Figure 20 has a zero joint likelihood suggesting the presence of a non-metallic object. This prediction is justified by observing that the magnetic sensor data is low while only the ultrasonic picks up the presence of an object.

\section{CONCLUSIONS}

This paper investigated the application of statistical algorithms in the form of Bayesian filter to enhance vehicle identification using ultrasonic and magnetic sensors. This research study utilized the knowledge gained by the authors in a previous study on the applicability of ultrasonic and magnetic sensor fusion for vehicle detection. This paper presented a detailed description of the procedure to formulate a two step 
prediction/correction based Bayesian filtering algorithm for both the ultrasonic and magnetic sensors. Statistical sensor models were developed for each type of sensor and individually utilized in the Bayesian filter algorithm. The results obtained showed a reduction in process noise and sensor anomalies that negatively influence the credibility of vehicle detection (as seen in Fig. 20). A joint Bayesian filter algorithm was then developed to facilitate sensor fusion. The results obtained clearly show the ability of the probabilistic approach to further enhance the prediction of object detection and discrimination capabilities of an ultrasonic-magnetic sensor fusion system. This study has also shed light on several other real-world applications of the ultrasonic-magnetic sensor combination such as its use in Smart toll booths, automation of parking lots, crash avoidance systems for trucks and passenger vehicles, etc. Future work will investigate these research venues.

\section{REFERENCES}

[1] A. Carullo, M. Parvis, "An ultrasonic sensor for distance measurement in automotive applications," Sensors Journal, IEEE,IEEE, vol.1, no.2, pp.143-147, Aug 2001.

[2] Kai-Tai Song; Chih-Hao Chen; Cheng-Hsien Chiu Huang, "Design and experimental study of an ultrasonic sensor system for lateral collision avoidance at low speeds," Intelligent Vehicles Symposium, 2004 IEEE , vol., no., pp. 647-652, 14-17 June 2004.

[3] MaxBotix, "LV-MaxSonar ${ }^{\circledR}-E Z 4^{\mathrm{TM}}$ High Performance Sonar Range Finder." MaxBotix ${ }^{\circledR}$ Inc. Jan. 2007. Feb. $2008<$ http://www.maxbotix.com/uploads/lv-maxsonarez4-datasheet.pdf $>$.

[4] J.E. Lenz: "A review of magnetic sensors," Proceedings of the IEEE , vol.78, no.6, pp.973-989, Jun 1990

[5] "Three-axis Magnetic Sensor Hybrid," Honeywell Magnetic Sensors, [http://www.magneticsensors.com/datasheets/hmc2003.pdf ].

[6] M. J. Caruso: "Applications of Magnetoresistive Sensors in Navigation Systems," Honeywell Inc.
[7] M. J. Caruso, L. S. Withanawasam: "Vehicle Detection and Compass Applications Using AMR Magnetic Sensors," Honeywell Inc.

[8] S. Y. Cheung, S. C. Ergen, and P. Varaiya: "Traffic Surveillance with Wireless Magnetic Sensors," Paper \#4779, University of California Berkeley, CA.

[9] R. Lao and D. Czajkowski: "Magnetoresistors for Automobile Detection and Traffic Control," SENSORS Mag., pp.70-73, April 1996

[10] T. Phan, B. W. Kwan, L. J. Tung: "Magnetoresistors for vehicle detection and identification," Systems, Man, and Cybernetics, 1997. 'Computational Cybernetics and Simulation'., 1997 IEEE International Conference on , vol.4, no., pp.3839-3843 vol.4, 12-15 Oct 1997

[11] "Application Note-AN218 Vehicle Detection Using AMR Sensors," Honeywell Magnetic Sensors. Aug. 2005. [http://www.magneticsensors.com/datasheets/an218.pdf].

[12] A. Shaffer, G. Aulakh, A. Getman, and R. Miller: "Vehicle Blind Spot Detection Using Anisotropic Magnetic Resistive Sensors," 2005 SAE International.

[13] A. Shaffer, G. Aulakh, A. Getman, and R. Miller. "Vehicle Sensors and Actuators (Part 1 \& 2)." SAE 2005 World Congress \& Exhibition, Apr. 2005, Detroit, MI, USA. SAE International. Vol. 2005-01-0456. SAE International, 2005. 53-58.

[14] S. Roussel, H. Porumamilla, C. Birdsong, P. Schuster, "Modeling of Vehicle Magnetic Footprint in 3-D Space for Type Detection"

[15] Pinheiro, Pedro, and Pedro Lima. Bayesian Sensor Fusion for Cooperative. Proc. of 8th Conference on Intelligent Autonomous Systems (IAS-8), 2004.

[16] Thurn, Sebastian, Wolfram Burgard, and Dieter Fox. Probabilistic Robotics. Cambridge: The MIT P, 2006.

[17]Fox, Dieter, Jeffery Hightower, Henry Kaus, Lin Liao, and Donald J. Patterson. Bayesian Techniques for Location Estimation. Proc. of 2003 Workshop on Location-Aware Computing.

[18] Koch, Karl-Rudolf. Introduction to Bayesian Statistics. New York: Springer, 2007. 